\title{
Whipple Triad Its Limitations in Diagnosis and Management of Hypoglycemia as a Co-morbidity in Covid-19 Diabetics and Diabetes Mellitus in General- A Review
}

\author{
Adegbenga Bolanle Ademolu \\ Medicine Department, Lagos State University Teaching Hospital, Ikeja, Lagos, Nigeria \\ Email address: \\ ademoluab@yahoo.com \\ To cite this article: \\ Adegbenga Bolanle Ademolu. Whipple Triad Its Limitations in Diagnosis and Management of Hypoglycemia as a Co-morbidity in Covid-19 \\ Diabetics and Diabetes Mellitus in General- A Review. International Journal of Diabetes and Endocrinology. Special Issue: Hypoglycemia \\ in Diabetes. Vol. 5, No. 2, 2020, pp. 23-26. doi: 10.11648/j.ijde.20200502.12
}

Received: February 25, 2020; Accepted: April 10, 2020; Published: May 29, 2020

\begin{abstract}
In 1938 Allen Oldfather Whipple (1881-1963) an American surgeon invented a triad of two clinical and one laboratory parameter to give the Whipple triad which had been in clinical use for decades and beneficial to perhaps millions of people worldwide with hypoglycemia as health challenges. The Whipple triad is characteristically presence of hypoglycemic symptoms, documentation of low blood sugar at the time of hypoglycemic symptoms, and reversal of these symptoms when the blood glucose level is restored to normal. Of note is the fact that over the years Whipple triad had been invaluable in diagnosing hypoglycemia in diabetics, but we should not lose sight of this singular fact that though good and useful for diagnostic purposes, Whipple triad has it's limitations as a diagnostic tool. With Corona virus disease 2019 (COVID 19) pandemic caused by severe acute respiratory distress syndrome coronavirus 2 (SARS CoV-2) affecting the immunosuppresed including diabetics, knowing the limitation of the diagnostic tool for diagnosing the hypoglycemia, a lifelong challenging issue in diabetics is a proactive way of recognizing a possible additional co-morbidity that if not recognized may further put the COVID-19 diabetic patient at risk. Though the issue of hypoglycemia in infected diabetic patient with COVID 19 has not been a major issue for now worldwide, the fact that asymptomatic carrier of COVID 19 diabetic patient could develop hypoglycemia makes it an important issue to be borne in mind by physicians. A google search was conducted in English by typing in the words Whipple, hypoglycemia, diabetes, probable hypoglycemia in pubmed. A literature review was done going through relevant articles. The limitations to Whipple triads were found in hypoglycemia with reversible neurological damage, hypoglycemia with irreversible neurological sequelae, hypoglycemia unawareness and in patients with rebound hypoglycemia. Similarly, Whipple triad limitation of application was found in unconscious patient from other causes other than hypoglycemia. Further limitations were also found in probable symptomatic hypoglycemia and in symptomatic hypoglycemia. Whipple triad has its limitation in medical practice which may be diverse and this have to be borne in mind by the clinician for a holistic approach to patient management especially during this COVID-19 pandemic and thereafter. The question to be addressed in the future remains of all hypoglycemic episodes occurring annually worldwide in diabetic and non diabetic subjects, how many can Whipple triad diagnose? In other words what is the sensitivity, specificity and predictive value of Whipple triad?
\end{abstract}

Keywords: Whipple Triad, Hypoglycemia, Diabetes Mellitus, Hypoglycemia Unawareness, Unconscious Patient, COVID 19, SARS CoV-2

\section{Introduction}

In the year1938Allen Oldfather Whipple (1881-1963) an American surgeon invented a triad of two clinical and one laboratory parameter to give the Whipple triad which had been in clinical use for decades and beneficial to perhaps millions of people worldwide with hypoglycemia as health challenges. Allen Oldfather Whipple is best known for the pancreatic cancer operation (pancreaticoduodenectomy) eponymously named the Whipple procedure of which he performed 37 during his career. Allen Oldfather Whipple described Whipple procedure in 1935 before describing Whipple triad three years later [1] in a publication titled "The surgical therapy of hyperinsulinism" [2]. 
The Whipple triad is characteristically presence of hypoglycemic symptoms, documentation of low blood sugar at the time of hypoglycemic symptoms, and reversal of these symptoms when the blood glucose level is restored to normal. Initially after invention, Whipple triad was thought to be indicative of potential hyperinsulinemia secondary to pancreatic insulinoma. Over the years and today it is now known that the application is not limited to patients with hyperinsulinemia from insulinoma, it is common knowledge that a major group involved in the application of Whipple triad are people living with diabetes who had hypoglycemia complicating their management at one time or the other especially type 1 diabetes mellitus patients who have hypoglycemia as a major issue to contend with in their day to day management of their diabetes mellitus.

Presently in the world, there is Corona virus disease 2019 (COVID 19) pandemic caused by severe acute respiratory distress syndrome coronavirus 2 (SARS-CoV-2) [3] Though the statistics is changing daily for now, presently, it has affected over 200 countries worldwide, affected all continents apart from Antarctica with over four million and seven hundred thousand people infected. This global figure is constantly changing and may be different when reader access this article. The World Health Organization and/or the center for disease control in each country may be contacted for latest updated figure.

Statistically, people with immunosuppression, comorbidity and the elderly are more prone to COVID 19. Among these immunosuppressed group are the diabetic patients [4]. Who already have the challenges of hypoglycemia as a co-morbidity complicating their management a times, Hence knowing the limitation of the diagnostic tool for diagnosing the hypoglycemia is a proactive way of recognizing a possible additional comorbidity that if not recognized may further put the COVID 19 diabetic patient at increased risk of morbidity and mortality. Though the issue of hypoglycemia in infected diabetic patient with COVID 19 has not been a major issue in literature for now, the fact that asymptomatic carrier of COVID 19 diabetic patient could develop hypoglycemia complicating management of diabetes makes it an important issue to be borne in mind by physicians.

Of note is the fact that over the years Whipple triad had been invaluable in diagnosing hypoglycemia in diabetes mellitus patients but we should not lose sight of this singular fact that though good and useful for diagnostic purposes, Whipple triad has it's limitations as a diagnostic tool which is the focus of this review article.

\section{Methods}

A google search was conducted in English by typing in the words Whipple, hypoglycemia, diabetes. probable hypoglycemia, Covid-19 in pubmed. A literature review was done going through relevant articles.

\section{Limitations of Whipple's Triad}

\subsection{Hypoglycemia with Reversible Neurological Damage}

Whipple triad does not have time frame attached to it. That is between time of presence of hypoglycemic symptoms which is also the time there is a demonstrable low blood sugar value and the time of resolution of neurologic symptoms with correction of hypoglycemia. In diabetics with hypoglycemia characterized by reversible neurological deficit, the clinical time of resolution of symptoms after correction of hypoglycemia is dependent on time it takes for the neurological deficit to resolve! This may be varied from one patient to the other. For instance headache is a reversible neurological symptom of hypoglycemia in some patient, but it is subjective and the time it takes to resolve depends on patients threshold. Seizure may manifest as reversible neurological symptoms of hypoglycemia, to abort the seizure in the acute stage, apart from giving dextrose infusion, benzodiazepines, anticonvulsants or barbiturates may be employed to abort the seizure due to metabolic derangement/condition precipitating it which in this case is hypoglycemia. Now full recovery from the underlying hypoglycemia will also be dependent on half life of the employed pharmacological agents used for the management of the seizure despite the fact that biochemical correction of hypoglycemia might have been achieved. This is an example of limitation of Whipple triad in the diagnosis and management of hypoglycemia.

\subsection{Hypoglycemia with Irreversible Neurological Sequelae}

It is well documented in literature that hypoglycemia in people living with diabetes mellitus can present with adrenergic and neurological symptoms [5-7]. It is also well known that the neurological symptoms of hypoglycemia can be reversible or irreversible. With respect to Whipple triad as a diagnostic tool, hypoglycemia with reversible neurological deficit can easily be diagnosed with very high positive predictive value. Unfortunately this same very high predictive value cannot be expected if the patient have irreversible neurological damage from previous or ongoing hypoglycemia especially if the irreversible neurological damage results in loss of consciousness. In such scenario only biochemical recovery of the hypoglycemia can be achieved without corresponding clinical response! This is a major limitation of Whipple triad! Clinicians not bearing this in mind may encounter a diagnostic dilemma after biochemical correction of hypoglycemia without corresponding clinical improvement if Whipple triad only is all the diagnostic criteria in focus to evaluate the suspected hypoglycemia. Irreversible neurological sequelae inducible by hypoglycemia can vary from coma to brain death these needs to be borne in mind in management of patients with hypoglycemia.

\subsection{Hypoglycemia Unawareness}

Hypoglycemia Unawareness [8-11] is one area of 
hypoglycemia complicating diabetes mellitus management that is challenging to both patients and healthcare provider as the apparently normal patient may be metabolically unstable glycemic-wise and in imminent danger of collapse. The fact that the patients is unaware of his hypoglycemic state or is asymptomatic makes one of the triad of Whipple that is symptoms compactible with hypoglycemia to be absent despite a demonstrable biochemical hypoglycemia. It therefore follows that Whipple triad is not applicable in diagnosing hypoglycemia characterized by hypoglycemia unawareness which is seen in long standing diabetes mellitus patients where the long standing diabetes mellitus is a risk factor for the hypoglycemia unawareness among other risk factors like degree of glycemic control, and the occurrence of repeated episodes of hypoglycemia [12, 13]! A situation where a asymptomatic COVID 19 carrier who has underlying diabetes mellitus also have hypoglycemia unawareness which is not recognized by Whipple triad is a potentially serious clinical scenario! Hence an high index of suspicion will be needed to diagnose the two condition if it co-exist in diabetics

\subsection{Rebound Hypoglycemia}

Whipple triad does not predict which patients will have rebound hypoglycemia at diagnosis or which have more chances of having rebound hypoglycemia at diagnosis. There is also the possibility that the initial hypoglycemic symptoms may not have fully resolved before a rebound hypoglycemia occurs. In such scenario, an overlap of symptoms may occur. There is need to research extensively into how long it takes for symptoms of hypoglycemia to resolve after correction of the hypoglycemia. The whipple triad is silent on this.

\subsection{Unconscious Patient from Other Causes Other than Hypoglycemia}

Loss of consciousness have varied etiology in medical practice [14], with possibly COVID 19 as an emerging cause [15], while a patient is unconscious, hypoglycemia may occur which may be biochemically detected. Diagnosing hypoglycemia in such patient clinically may create diagnostic challenge as the adrenergic and neurologic symptoms like excessive hunger, general body weakness, palpitation, sweating, tremor, dizziness, cannot be complained by the unconscious patient. Hence one of the triad of Whipple that is presence of hypoglycemic symptoms at the time of documentation of biochemical hypoglycemia will be absent. Furthermore since hypoglycemia was not the primary cause of loss of consciousness, correction of the hypoglycemia with documented biochemical recovery without correction of the primary cause of the unconsciousness may not necessarily lead to clinical recovery from unconsciousness. This is another limitation of Whipple triad.

\subsection{Probable Symptomatic Hypoglycemia}

Probable symptomatic hypoglycemia is typical hypoglycemic symptoms responding to self-treatment but not confirmed by biochemical documentation but presumably caused by plasma glucose less than or equal to $70 \mathrm{mg} / \mathrm{dL}$. This is commonly seen in outpatient diabetics than hospitalised diabetics. I have come across many diabetics who by virtue of been used to their disease condition can tell when they are hypoglycemic clinically without waiting to check their blood sugar level. Some go on to correct this without checking objectively their hypoglycemic status by checking their blood sugar level. This type of hypoglycemia may truly have symptoms of hypoglycemia which is relieved by correction with carbohydrate ingestion but the absent of laboratory value in diagnosis makes Whipple triad not applicable in this clinical form of hypoglycemia [16, 17].

\subsection{Symptomatic Hypoglycemia}

The Whipple triad does not give insight on rate of recovery from symptomatic hypoglycemia. It does not give room for clinicians to recognize hypoglycemia that resolves while giving glucose before the blood sugar level is biochemically restored back to normal values just as it is silent on how long it takes for hypoglycemic symptoms that did not resolve below normal biochemical blood glucose value to resolve after blood sugar is restored to normal. The rate of recovery in these two scientific scenario depends on many factors which may includes co-morbidity, biochemical level of hypoglycemia at diagnosis, route of glucose administration during correction of hypoglycemia, dose or amount of glucose given, drugs patient is on at the time of diagnosing symptomatic hypoglycemia (e.g sulfonylureas, meglitinides, type of insulin whether long acting or short acting, etc.) [18-20].

\section{Discussion}

Whipple triad is useful but with limitation in a variety of clinical scenario as seen above. Despite technological advancement like widespread presence of glucose meter devise and the emerging use of continous glucose monitoring device that makes biochemical measurement of blood sugar easier than what it used to be many decades ago when Whipple described his triad of diagnosing hypoglycemia, the application of Whipple triad is still suffering setback in the present age because of its limitation. This fact has to be noted carefully in diagnosing hypoglycemia in COVID-19 infected diabetic patients irrespective of their clinical features. With the whole world faced with the reality of COVID 19 pandemic and spread with no definitive therapy in view for now coupled with the effect it has on endocrinology and diabetics in particular, attention must be paid to modifiable and preventable co-morbidities like hypoglycemia in affected diabetics with COVID 19 infection whether symptomatic or asymptomatic now and in the future.

Recognizing hypoglycemia at a time like this is crucial since diabetics are already immunosuppressed and diabetes is usually associated with co-morbidity. Reducing the number of comorbidity by appropriately diagnosing hypoglycemia among other co-morbidities of diabetes perhaps will help to improve outlook or prognosis in diabetics with COVID 19 infection. 


\section{Future Direction}

Specificity, sensitivity and predictive value of Whipple triad in diagnosing hypoglycemia complicating diabetes mellitus management should be looked into.

The prevalence of Covid-19 in diabetics in each country and worldwide should be determined and compared in type 1 and type 2 diabetes mellitus. Covid-19 in diabetics should be studied in terms of gender distribution, severity in different diabetic age groups and so on, Covid-19 in diabetics can also be studied comparatively with other infective conditions found in diabetic patients. The prognosis of hypoglycemia occurring in asymptomatic COVID 19 carrier with diabetes mellitus should be studied and compared to prognosis of asymptomatic COVID 19 carrier in other immunosuppresed disease condition and also in the general population.

\section{Limitations of the Study}

Search was done in English. Region by region search of the world was not done. The exact value of Covid-19 prevalence could not be given since it is dynamically changing worldwide.

\section{Conclusion}

Whipple triad has its limitation in medical practice which may be diverse and this have to be borne in mind by the clinician for a holistic approach to patient management especially in this new era of COVID-19 pandemic. The question to be addressed in the future remains of all hypoglycemic episodes occurring annually worldwide in diabetic and non diabetic subjects, how many can whipple triad diagnose? In other words what is the sensitivity, specificity and predictive value of Whipple triad?

\section{Conflict of Interest}

None.

\section{References}

[1] Dr Chloe Roy. Allen Oldfather Whipple. Life in the fastlanelast update May 24, 2019.

[2] Allen Oldfather Whipple. The surgical therapy of hyperinsulinism. J Internat Chir3: 237-276 (1938).

[3] Marco Cascella; Michael Rajnik; Arturo Cuomo; Scott C. Dulebohn; Raffaela Di Napoli. Features, Evaluation and Treatment Coronavirus (COVID-19).

[4] Lei Fang, George Karakiulakis, *Michael Roth. Are patients with hypertension and diabetes mellitus at increased risk for COVID-19 infection? The Lancet Respiratory Medicine Volume 8, ISSUE 4, Pe21, April 01, 2020.
[5] Auer RN. Hypoglycemic brain damage. Metab Brain Dis. 2004 Dec; 19 (3-4): 169-75.

[6] Roger F Butterworth. Hypoglycemic Encephalopathy. American Society for Neurochemistry. 1999.

[7] Nana Esi Kittah et al. MANAGEMENT OF ENDOCRINE DISEASE: Pathogenesis and management of hypoglycemia.., European Journal of Endocrinology, 2017.

[8] Philip E. Cryer, Lloyd Axelrod, Ashley B. Grossman, Simon R. Heller Victor M. Montori, Elizabeth R. Seaquistet al. Evaluation and Management of Adult Hypoglycemic Disorders: An Endocrine Society Clinical Practice Guideline. The Journal of Clinical Endocrinology \& Metabolism, Volume 94, Issue 3, 1 March 2009, Pages 709-728.

[9] Martín-Timón I, Del Cañizo-Gómez FJ. Mechanisms of hypoglycemia unawarenessand implications in diabetic patients. World J Diabetes. 2015; 6 (7): 912-926. doi: 10.4239/wjd.v6.i7.912.

[10] Samson WK, Stein LM, Elrick M, et al. Hypoglycemia unawareness prevention: Targeting glucagon production. Physiol Behav. 2016; 162: 147-150. doi: 10.1016/j.physbeh.2016.04.012.

[11] Bakatselos SO. Hypoglycemia unawareness. Diabetes Res Clin Pract. 2011 Aug; 93 Suppl 1: S92-6. doi: 10.1016/S01688227(11)70020-1.

[12] Andreas Fritsche, Michael Stumvoll, Hans U. Häring, John E. Gerich Reversal of Hypoglycemia Unawareness in a LongTerm Type 1 Diabetic Patient by Improvement of $\beta$-Adrenergic Sensitivity after Prevention of Hypoglycemia. The Journal of Clinical Endocrinology \& Metabolism, Volume 85, Issue 2, 1 February 2000, Pages 523-525.

[13] Kalra S, Mukherjee JJ, Venkataraman S, et al. Hypoglycemia: The neglected complication. Indian J Endocrinol Metab. 2013; 17 (5): 819-834. doi: 10.4103/2230-8210.117219.

[14] M Hassan Murad, Fernando Coto-Yglesias, et al. Drug-Induced Hypoglycemia: A Systematic Review. The Journal of Clinical Endocrinology and Metabolism March 200994 (3): 741-5.

[15] Sharifi-Razavi A, Karimi N, Rouhani N, COVID 19 and Intra cerebral hemorrhage: Causative or Coincidental, New Microbes and New Infections.

[16] Javier Morales, MD, a Doron Schneiderb. Hypoglycemia. The American Journal of Medicine, Vol 127, No 10 A, October 2014.

[17] Seaquist ER, Anderson J, Childs B, et al. Hypoglycemia and diabetes: a report of a workgroup of the American Diabetes Association and the Endocrine Society. Diabetes Care. 2013; 36 (5): 1384-1395.

[18] International Hypoglycaemia Study Group*. Minimizing Hypoglycemia in Diabetes. Diabetes Care 2015 Aug; 38 (8): 1583-1591.

[19] Philip Mathew; Deepu Thoppil. Hypoglycemia. Treasure Island (FL): Stat Pearls Publishing, 2020 Jan.

[20] D. Clayton et al. Hypoglycemia. / Can J Diabetes 37 (2013) S69eS71. 